\title{
Robust Range Assignment Strategy for Wireless Sensor Networks"
}

\author{
Xuejian Zhao, Xiaojun Wang, Bin Xu \\ College of Internet of Things, Nanjing University of Posts and Telecommunications, Jiangsu, china \\ \{zhaoxj \& wangxj \& xubin\}@njupt.edu.cn
}

\begin{abstract}
The RA (Range Assignment) strategy is an art of setting nodes' transmitting ranges to generate a network with desired properties and at the same time reducing node energy consumption and/or increasing network capacity. In this paper, a distributed RA strategy RRAS (Robust Range Assignment Strategy) is proposed. It shows mainly three advantages to most previously proposed topology control algorithms. First, it is strictly local and does not require node position information. Second, the resulting topologies of RRAS algorithm under different coefficients are available for various application scenarios, such as energy-efficient applications, real-time performance applications and so on. Third, each node can adjust the coefficient dynamically to maintain the network connected for a longer time. Analysis and simulation results are still given to demonstrate the correctness and effectiveness of our proposed algorithm.

Index Terms - Wireless sensor networks, range assignment, topology control, robustness, dynamic coefficient adjustment.
\end{abstract}

\section{I . Introduction}

Wireless sensor networks are formed by a large number of power-conscious wireless-capable sensors without the support of pre-existing infrastructure, usually by unplanned deployment. As is known, it is better to communicate using short, multi-hop paths between the sender and the receiver in wireless sensor networks from both the energy-consumption point of view and the network capacity point of view. Topology control via per-node transmission power adjustment has been shown to be effective in extending network lifetime and increasing network capacity.

Topology control is the art of coordinating nodes' decisions regarding their transmitting ranges, in order to generate a network with the desired properties (e.g. connectivity, scalability, robustness) while reducing node energy consumption and/or increasing network capacity [1], which has been a subject of intensive research in recent years. Many different topology control mechanisms are proposed. Paolo Santi organized these diverse topology control approaches into two categories: homogeneous CTR (Critical Transmitting Range) and nonhomogeneous topology control [1]. In the former case, all the network nodes must use the same transmitting range, and the topology control problem reduces to the simple problem of determining the minimum value of the transmitting range such that a certain networkwide property is satisfied. In nonhomogeneous topology control, nodes are allowed to choose different ranges. Depending on the type of information that is used to compute the topology, nonhomogeneous topology control is further classified into three categories: location based, direction based and neighbor based.

The CTR for connectivity in stationary and mobile networks are much investigated in recent years, and it is found that determining the CTR for all the nodes in a distributed way is impossible [2]. However, in many scenarios, sensor nodes can change the transmit power level. Consequently, we could consider the problem of determining a set of power level assignments that generates a connected communication graph while at the same time minimizing the energy consumption and maximizing the network capacity. This problem is known in the literature as the RA (Range Assignment) problem which was first studied by Kirousis et al. [3].

\section{II . The Range Assignment Problem}

Different from the definition given by Kirousis et al [3], the RA problem in this paper is defined as follows.

Definition 1 RA (Range Assignment) Problem: Let $\mathrm{N}$ be a set of nodes in the d-dimensional space, with $\mathrm{d}=1,2,3$. Determine the transmitting range $r_{i}(i \in[1, N])$ for every node to generate a network with the desired properties (e.g. connectivity, scalability, robustness) while reducing node energy consumption and increasing network capacity.

As mentioned above, minimizing the nodes' transmitting range is an effective manner to reduce node energy consumption and increasing network capacity. Thus, RA can be informally stated as the problem of finding all the nodes' minimal range assignment to generate a connected communication graph with other desired properties in specifically application scenarios.

In a certain sense, the RA problem can be seen as a generalization of the problem of determining the CTR, where the constraint that all the nodes have the same transmitting range is dropped. Obviously, dropping this constraint considerably increases the complexity of finding the optimal solution.

Kirousis et al. present an algorithm to find the optimal solution for the RA problem in case of one-dimensional networks. At the same time, they proved the NP-hardness of

\footnotetext{
* This work is partially supported by the National Natural Science Foundation of China Grant \#61101104 to Xu Liu and 61100213 to Kun Wang, the Natural Science Foundation of the high school in Jiangsu Province Grant \#12KJB520012, and the Fund of Nanjing University of Posts and Telecommunications Grant \#NY211050.
} 
finding the optimal solution to RA problem in threedimensional networks [3]. Later on, Clementi et al. proved that the RA problem remains NP-hard in case of two-dimensional networks also [4]. In the RA problem, the nodes in general have different transmitting ranges, unidirectional links might occur, and this can be essential for ensuring strong connectivity of the networks. However, most routing protocols and MAC protocols are based on the implicit assumption that wireless links must be bidirectional. The reason motivated researches to investigate restricted versions of the RA problem, where certain symmetry constraints are imposed on the communication graph. For instance, Blough et al. defined and investigated the WSRA (Weakly Symmetric Range Assignment) problem and SRA (Symmetric Range Assignment) problem [5]. Since solving WSRA or SRA in two and three dimensional networks is NP-hard, Althaus et al. turned to study approximation algorithms for WSRA and SRA [6-11].

In RA approaches, it is usually assumed that the most accurate information about node positions is known. This information is either used by a centralized authority to compute a set of transmitting range assignments that optimizes a certain measure, or it is exchanged between nodes and used to compute an approximately optimal topology in a fully distributed manner. In addition, nearly all RA strategies emphasize only minimizing the nodes' range assignment values for energy conservation and network capacity. Consequently, the resulting topology is more and more sparse. As a result, the network is easily to be separated when some nodes deplete their energy or suffer external attacks. The robustness of the resulting topology and how to maintain the network connectivity have not been considered considerably yet

Therefore, in this paper, a novel RA strategy RRAS (Robust Range Assignment Strategy) without requiring node location information is proposed. It works in a fully distributed manner, and the resulting topologies of RRAS under different coefficients are available for different application scenarios, such as energy-efficient applications, real-time performance applications and so on. Further more, it can strike a balance between sparseness and robustness by adjusting the coefficient dynamically to maintain the network connectivity. In order to verify the correctness and effectiveness of the proposed algorithm, the performance of RRAS is compared with an outstanding topology control algorithm XTC which was presented by Wattenhofer and Zollinger [12] by simulation.

\section{A. Preliminary Assumptions and Definitions}

In this section, the RRAS algorithm will be described. Before presenting the algorithm, we need some preliminary assumptions and definitions. The assumptions are as follows:

(1) A set of $N$ nodes $V=\left\{v_{1}, v_{2}, \ldots, v_{N}\right\}$, are uniformly distributed in a two- or three-dimensional space, and all the nodes are homogeneous and remain stationary all the time.

(2) Each node has a unique ID.

(3) The wireless channel is symmetric and obstacle-free, which means the path loss model is the same for all the network nodes and the shadowing effects are not considered. Consequently, transmitting range and transmit power level are equivalent concepts.

(4) There exists an underlying MAC protocol supporting for this algorithm, which means we do not need to take into account the interference and so on.

Let $G=(V(G), E(G))$ be the network topology graph, where $V(G)$ and $E(G)$ are the node set and edge set respectively. We define the ordered pair $(u, v)$ as an edge from node $u$ to node $v$, and the Euclidean distance between them is $\operatorname{dist}(u, v)$, such that $(u, v) \neq(v, u)$ and $\operatorname{dist}(u, v)=\operatorname{dist}(v, u)$. Let $r_{\max }$ denote the maximum transmission range, so every node's range assignment value should not exceed $r_{\max }$.

Definition 2 Weight of Edge ( $W_{u v}$ ): The weight of edge $W_{u v}$ can reflect the quality of the link between node $u$ and node $v$, such as Euclidean distance, signal attenuation or packet arrival rate and so on.

Definition 3 Original Topology Graph ( $\left.G_{o}\right)$ : The Original Topology Graph denotes the network on condition that all the nodes' transmitting range is $r_{\max }$.

Definition 4 Target Topology Graph $\left(G_{t}\right)$ : The Target Topology Graph denotes the network by pruning the energyinefficient and capacity-inefficient edges through some special algorithms. The Target Topology Graph can be represented by $G$ with the topology algorithm name as a subscript.

Definition 5 Physical Neighbor Set: The Physical Neighbor Set of node $u$ is $P N S(u)=\left\{v \mid \operatorname{dist}(u, v) \leq r_{\max }, v \in V(G)\right\}$.

Definition 6 Logical Neighbor Set: The Logical Neighbor Set of node $u$ is $L N S(u)=\left\{v \mid \operatorname{dist}(u, v) \leq r_{u}, v \in V(G)\right\}$, where $r_{u}$ is node $u$ 's range assignment value. That is to say there exists an edge $(u, v)$ in the target topology graph if and only if $\operatorname{dist}(u, v) \leq r_{u}$, and $E\left(G_{t}\right)=\left\{(u, v) \mid \operatorname{dist}(u, v) \leq r_{u}, u \in V(G)\right.$, $v \in \operatorname{LNS}(u)\}$.

\section{B. The RRAS Algorithm}

The RRAS algorithm consists of five main steps as follows:

(1) Each node in the network broadcasts a Hello message using the maximum transmitting range $r_{\max }$, and the Hello message includes the node ID. Consequently, the Hello message could be received by all the nodes in the sender's Physical Neighbor Set. That is to say, every node for example $u$ obtains $P H S(u)$.

(2) Each node computes a total order over all its physical neighbor nodes according to RSSI (Received Signal Strength Indication), for instance the total order computed by node $u$ is denoted as $\prec_{u}$. On the preliminary assumptions the Euclidean distance of the nodes can be approximately computed according to the RSSI value. That is to say, if node $w$ lies in front of node $v$ in $\prec_{u}$, denoted as $w \prec_{u} v$, then we can infer 
$\operatorname{dist}(u, w)<\operatorname{dist}(u, v)$. In this paper, the quality of the links stands for the Euclidean distance between the nodes.

(3) Each node broadcasts its computed order to all its physical neighbors and stores the orders received from its physical neighbors.

(4) Each node selects nodes from its physical neighbors to compose the Logical Neighbor Set in this step. For this purpose a node $u$ traverses $\prec_{u}$ according to the link quality in descending order. Informally speaking, good neighbors are considered first, worse ones later. When node $v$ is considered, it will not be included in $L N S(u)$ if there is a node $w$ satisfies conditions: $w \prec_{u} v, \quad w \prec_{v} u$ and $f\left(W_{u w}, W_{v w}\right)<g\left(W_{u v}\right)$. Otherwise the node $v$ is selected as an element of $\operatorname{LNS}(u)$. In this paper, we let $f\left(W_{u w}, W_{v w}\right)=\operatorname{dist}(u, w)^{\alpha}+\operatorname{dist}(v, w)^{\alpha}$ and $g\left(W_{u v}\right)=\lambda \operatorname{dist}(u, v)^{\alpha}$, where $\alpha$ and $\lambda$ are adjustable coefficients.

(5) Each node selects the transmit power level which can just cover all the nodes in $\operatorname{LNS}(u)$. That is to say, node $u$ can regard $\operatorname{dist}(u, v)$ as its range assignment value, where node $v$ has a bigger $W_{u v}$ than other nodes in $L N S(u)$.

The RRAS algorithm works in a fully distributed manner and only local information is needed. The pseudo-code for node $u$ is:

1: Broadcasts a Hello message using the maximum transmitting range $r_{\max }$;

2: Receives the Hello messages from all the nodes in $P N S(u)$;

3: Computes the total order $\prec_{u}$ over all its physical neighbor nodes according to RSSI of the Hello messages;

4: Broadcasts the total order $\prec_{u}$ and stores the orders received from its physical neighbors;

5: Let $\operatorname{LNS}(u):=\{\}, \operatorname{LNS}(u):=\{\}$;

6: While $\left(\prec_{u}\right.$ contains unprocessed neighbors $)\{$

7: $v:=$ unprocessed neighbor node in $\prec_{u}$ with the best link quality;

8: If $\left(\exists w \in L N S(u) \cup L N \tilde{S}(u): w \prec_{v} u \wedge f\left(W_{u w}, W_{v w}\right) \leq g\left(W_{u v}\right)\right.$

9: then $\operatorname{LNS}(u)=\operatorname{LNS}(u) \cup\{v\}$

10: else $\operatorname{LNS}(u)=\operatorname{LNS}(u) \cup\{v\} ;\}$;

11: Finally $r_{u}=\operatorname{dist}(u, v)$, where $v \in L N S(u)$ and for $\forall w \in L N S(u), \operatorname{dist}(u, v) \geq \operatorname{dist}(u, w)$.

\section{IV . Simulation Analysis}

In order to verify the performance of RRAS, the algorithm is implemented by OMNET++ and some experiments are carried out. In all the simulations, we assume that 60 nodes (No.0 No.59) are dispersed in an $800 \mathrm{~m} * 500 \mathrm{~m}$ rectangular district. And all the nodes have the same maximum transmitting range 250 meters. The initial topology $G_{o}$ and resulting topology $G_{X T C}$ and $G_{R R A S}$ are as Figure 1 shows. We can observe that, if $\lambda$ increases gradually, the resulting topology of RRAS becomes more and more sparse. When $\lambda=1.5$, the resulting topology $G_{R R A S}$ is nearly as sparse as $G_{X T C}$.

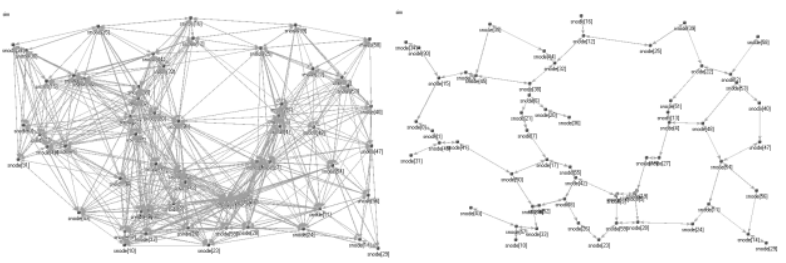

a. $G_{o}$

b. $G_{X T C}$

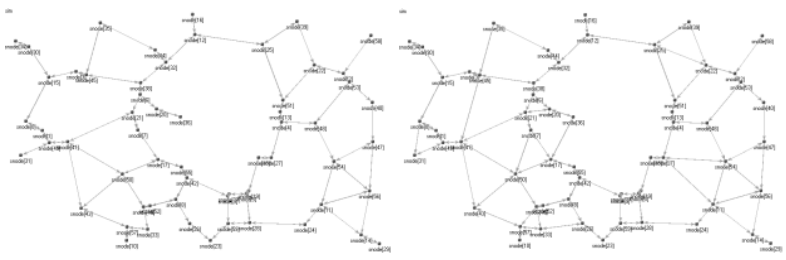

c. $G_{R R A S}, \lambda=1.5$

d. $G_{R R A S}, \lambda=1.2$

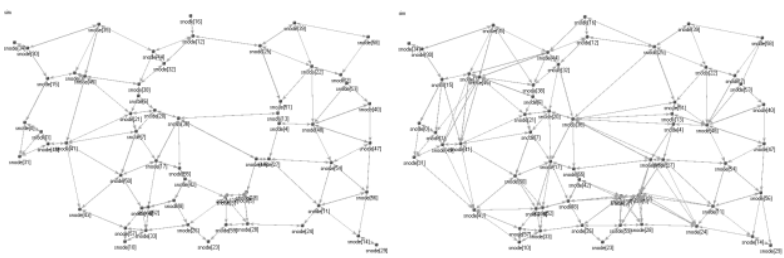

$$
\text { e. } G_{R R A S}, \lambda=1
$$

f. $G_{R R A S}, \lambda=0.8$

Fig.1 a. The initial topology graph $G_{o}$;

b. the resulting topology of XTC algorithm $G_{X T C}$;

c. the resulting topology of RRAS algorithm $G_{R R A S}$ when $\lambda=1.5$;

d. the resulting topology of RRAS algorithm $G_{R R A S}$ when $\lambda=1.2$;

e. the resulting topology of RRAS algorithm $G_{R R A S}$ when $\lambda=1$;

f. the resulting topology of RRAS algorithm $G_{R R A S}$ when $\lambda=0.8$.

Figure 2 shows the range assignment values of the nodes in the resulting topology graph of XTC algorithm and RRAS algorithm when $\lambda$ varying from 0.8 to 1.5 . We can observe in Figure 2 that, the range assignment values of the nodes reduce gradually when the coefficient $\lambda$ varies from 0.8 to 1.5 . Further more, the range assignment values of the RRAS algorithm are close to the values obtained from the excellent XTC algorithm proposed by Wattenhofer and Zollinger when $\lambda$ is set to 1.5. Consequently, if the most important property considered by the topology control algorithm is energy conservation or network capacity, we should set $\lambda$ as big as we can as long as the resulting topology satisfies the other properties. Of course, it should not exceed 2 according to our previous analysis. 


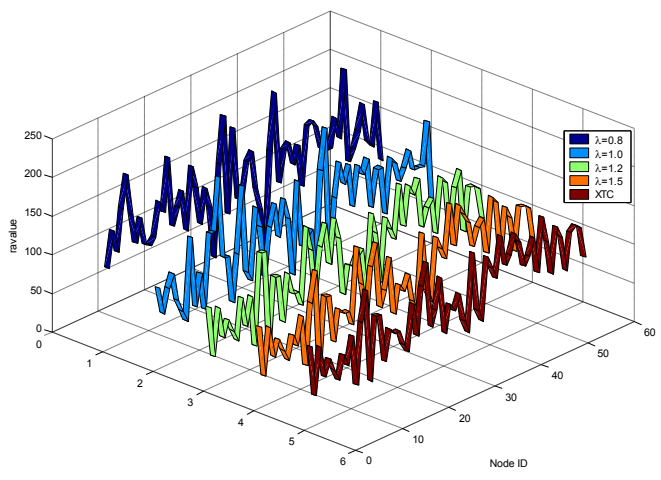

Fig.2 The comparision of the range assignment values.

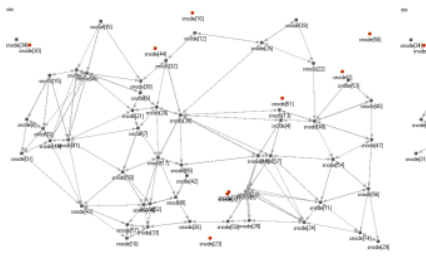

a. $G_{R R A S}, \lambda=0.8$

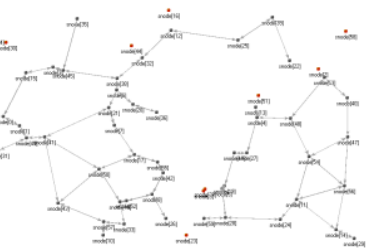

b. $G_{R R A S}, \lambda=1.5$

Fig.3 a. the topology $G_{R R A S}(\lambda=0.8)$ when a certain number of nodes exhaust their energy;

b. the topology $G_{X T C}$ when a certain number of nodes exhaust their energy

As is known, in the field of topology control in wireless sensor networks, robustness is the ability of maintaining connectivity when a few of the nodes in the network exhaust their energy or be attacked. It is obvious that a sparse topology, whose nodes have a small average degree, is more easily to be isolated. For instance, we assume a certain number of nodes are dead in our experiments. As Figure 3 shows, $G_{R R A S}(\lambda=1.5)$ is separated into two large isolated components. However, $G_{R R A S}(\lambda=0.8)$ remains connected except a marginal node. Overall, $G_{R R A S}$ is more robust than $G_{X T C}$ no matter what the coefficient $\lambda$ is. Of course, if we set $\lambda$ much smaller within its domain, the resulting topology will be more robust.

\section{Conclusion}

In this paper, a distributed RA strategy RRAS is proposed. We describe the RRAS algorithm in detail and discuss the properties of the algorithm. Simulation results show that
RRAS algorithm can satisfy different application requirements such as energy-efficient, small transmission delay, robustness and so on by adopting a proper $\lambda$. In future research, a proper routing protocol based on the resulting topology of RRAS algorithm with dynamic coefficient adjustment strategy should be proposed for a further analysis of the network performance.

\section{References}

[1] Paolo Santi, "Topology Control in Wireless Ad Hoc and Sensor Networks," Wiley, 2005, pp.31-32.

[2] Narayanaswamy S, Kawadia V, Sreenivas R and Kumar P, "Power control in ad hoc networks: theory, architecture, algorithm and implementation of the compow protocol," Proc. European Wireless 2002, Florence, pp. 156-162.

[3] Kirousis L, Kranakis E, Krizanc D and Pelc A, "Power consumption in packet radio networks," Theoretical Computer Science, vol. 243, pp. 289-305, 2000.

[4] Clementi A, Penna P and Silvestri R, "Hardness results for the power range assignment problem in packet radio networks," Proc. 2nd International Workshop on Approximation Algorithms for Combinatorial Optimization Problems (RANDOM/APPROX'99), Berkeley, CA, 1999, pp. 197-208.

[5] Blough D, Leoncini M, Resta G and Santi P, "On the symmetric range assignment problem in wireless ad hoc networks," Proc. IFIP Conference on Theoretical Computer Science, Montreal, 2002, pp. 71-82.

[6] Althaus E, Calinescu G, Mandoiu I, Prasad S, Tchervenski N and Zelikovsky A, "Power efficient range assignment in ad hoc wireless networks," Proc. IEEE WCNC 03, New Orleans, LA, 2003, pp. 18891894.

[7] Tong Z, Haenggi M. Convergence of Power Control Algorithms for Wireless Networks with and without Fading. IEEE Transactions on Vehicular Technology, 2012:1-27.

[8] Juan Luo, Chen Pan, Renfa Li, and Fei Ge. Power Control in Distributed Wireless Sensor Networks Based on Noncooperative Game Theory, International Journal of Distributed Sensor Networks, vol. 2012, Article ID 398460, 10 pages, 2012. doi:10.1155/2012/398460.

[9] Wayes Tushar, David Smith,Tharaka A. Lamahewa, Jian Zhang. Noncooperative power control game in a multi-source wireless sensor network. Communications Theory Workshop (AusCTW), Australian, 2012:43-48

[10] Shang Jin, Jingqi Fu, Liming Xu. The Transmission Power Control Method for Wireless Sensor Networks Based on LQI and RSSI. In: Asia Simulation Conference 2012(AsiaSim 2012), Shanghai, China, October 27-30, 2012:37-44.

[11] Basavaraj S. Mathapati, Siddarama R. Patil, V.D. Mytri. Distributed power control with energy efficient forwarding routing protocol for wireless sensor networks. International Journal of Information Technology and Knowledge Management, 2012,5(2),387-392.

[12] Roger Wattenhofer and Asron Zollinger, "XTC: A practical topology control algorithm for ad-hoc and sensor networks," in 4th International Workshop on Algorithms for Wireless, Mobile , Ad Hoc and Sensor Networks (WMAN), 2004.

[13] Rappaport T, "Wireless Communications: Principles and Practice," Second Edition, Prentice Hall, Upper Saddle River, NJ, 2002. 